\title{
HISTORIA
}

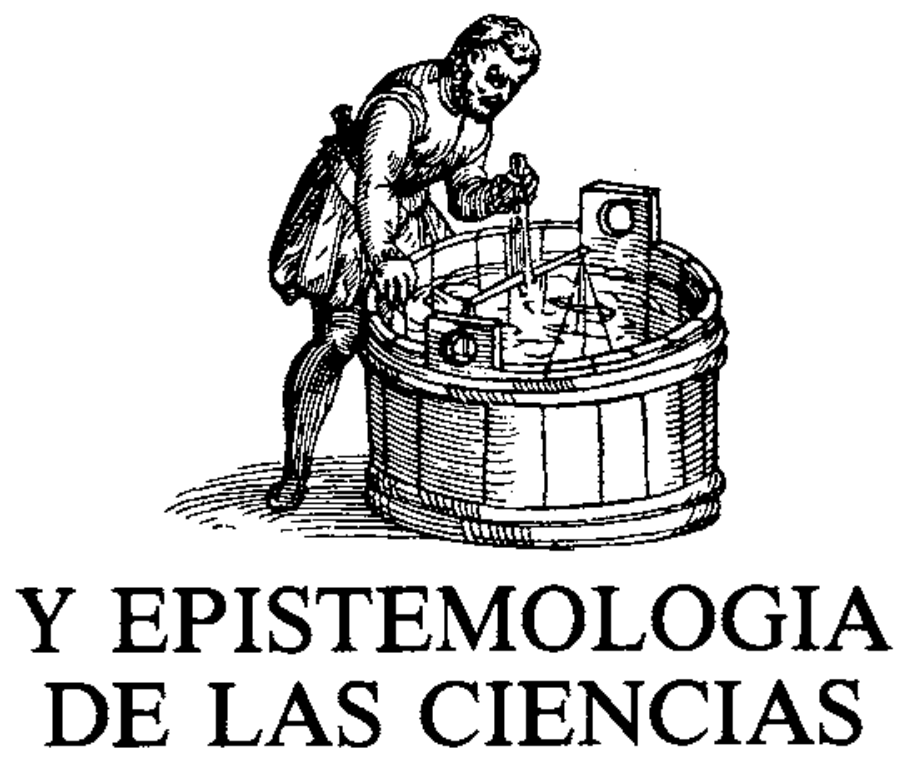

\section{EL CONCEPTO DE GEOSINCLINAL TRAS LA REVOLUCIÓN MOVILISTA: OTRO DESAJUSTE ENTRE LA CIENCIA ACTUAL Y LA CIENCIA EN EL AULA}

ANGUITA, $F$.

Facultad de Ciencias Geológicas. Universidad Complatense.

\section{SUMMARY}

A revision of Geology and Physical Science textbooks widely used at high-school level shows that the geosyncline idea (in itself or as a part of plate tectonics theory) is frequently presented as a tenable hypothesis on mountain origins. As this is not a scientifically acceptable position any more, a great deal of confusion is probably being created among both students and teachers without very solid geologic foundations.

\section{INTRODUCCIÓN}

«Sin embargo, las nuevas ideas sobre extensión del fondo oceánico, y tectónica de placas constituyen un nuevo paradigma que amenaza con reemplazar, o quizá ya ha reemplazado, al antiguo, a saber, el concepto de 
geosinclinal, que ha servido a generaciones de geólogos durante más de un siglo». El oceanógrafo chino Kenneth H. Hsü escribió esas cautas palabras en 1972, sólo diez años después de la única revolución que se ha producido en la ciencia después de que Kuhn escribiese La estructura de las revoluciones cientificas. Hoy, casi veinte años después de Hsii, demasiados textos de Ciencias Naturales o Geología destinados al nivel medio siguen reflejando una profunda desorientación en este tema, que es clave para comprender la Tierra. Hay diversas variantes: en unas, los geosinclinales aparecen como el concepto central, y la tectónica de placas como una especie de añadido; en otras sucede al revés, pero la apostilla final parece indicar que el geosinclinal es perfectamente integrable en el movilismo moderno, como si todo lo que hubiese cambiado fuese que «ahora» los geosinclinales se desplazan lateralmente. Los profesores que imparten Geología en este nivel, y que en general son biólogos, piden insistentemente una clarificación sobre este aspecto nuclear de la Geología.

Esta reevaluación del concepto de geosinclinal se ha producido hace años en otros países: primero en revistas científicas, y luego en publicaciones dedicadas a los docentes de Geología. Aunque con ocho o diez años de retraso, reproducir aquí los temas claves de este debate, y añadir incluso un punto de vista actual, parece algo necesario si queremos que a nivel pedagógico la Nueva Geología suponga un avance auténtico y no la confusión de ideas que surge de una revolución mal digerida.

\section{EL CONCEPTO DE GEOSINCLINAL (1859-1964)}

La historia, muy repetida, de esta idea científica se remonta a mitad del siglo XIX. James Hall, un paleontólogo que trabajaba para el Servicio Geológico del Estado de Nueva York, tuvo la idea de que, si la estructura que había observado en los Apalaches era generalizable, las cadenas de montañas eran precedidas por el depósito de una enorme cantidad de sedimentos, al menos un orden de magnitud mayor que en las zonas estables. Para contener esta masa de sedimentos Hall imaginó un surco gigantesco, pero como le parecía inaceptable que el Este de Norteamérica hubiese estado nunca cubierto por diez o doce kilómetros de agua, propuso que el fondo del surco se iba hundiendo bajo el peso de los propios sedimentos. A determinada profundidad, la base de éstos se metamorfizaría y fundiría, y movimientos en la vertical causados esencialmente por el ascenso del magma terminarían por plegar los sedimentos.

Este esquema básico fue propuesto por Hall en 1859 , precisamente el año en que se publicaba El origen de las especies. No deja de ser irónico que el mismo año en que en las ciencias de la vida se proponía el que iba a ser su paradigma más explicativo, en las ciencias de la Tierra se inaugurase un siglo de confusión. Este concepto primitivo sufrió innumerables complicaciones: se hizo intervenir la contracción terrestre, se crearon geoanticli- nales que se elevaban para compensar el hundimiento de los geosinclinales, se propuso el deslizamiento gravitacional de grandes masas de sedimentos y se adoptó una impresionante terminología de raíces griegas: ortogeosinclinal o geosinclinal real, parageosinclinal of also geosinclinal, eugeosinclinal o geosinclinal completo, miogeosinclinal o geosinclinal menor... Esta tendencia a acuñar neologismos desembocó en lo que Kelling (1981) ha denominado «frenesí etimológico», y alcanzó su culminación con Kay (1951), quien definió más de treinta tipos diferentes de geosinclinales sólo para explicar las cordilleras norteamericanas. Al cabo de casi un siglo, la idea inicial se había convertido en un macroconcepto, el «ciclo geotectónico», bajo el que se aspiraba a englobar la evolución que llevaría a una cuenca sedimentaria a transformarse en una cadena de montañas. El ciclo geotectónico comprendía tres fases: fase geosinclinal (acumulación de sedimentos), fase tectogenética (deformación y plutonismo) y fase orogénica (elevación de la cadena, vulcanismo y colapso gravitacional). Aunque hubo muy distintas variantes, el concepto de ciclo geotectónico tuvo dos bases fundamentales:

1) El geosinclinal sufre grandes movimientos verticales (que a nivel sedimentario se expresan como subsidencia).

2) La deformación compresiva del geosinclinal es el acontecimiento clave de su evolución; esta compresión se produce simultánea y universalmente en todos los geosinclinales del planeta (las tablas de fases de plegamiento son un residuo de esta idea).

La contrapartida actualista de los geosinclinales del pasado se hallaba en ejemplos como el de la costa atlántica norteamericana, citado por Dietz (1963), y en la que los sedimentos de la plataforma continental corresponderían a un miogeosinclinal, y los del talud continental a un eugeosinclinal.

\section{LA ETAPA DE TRANSICIÓN (1964-1975)}

A partir de marzo de 1964, en el transcurso del simposio sobre deriva continental organizado en Londres por la Royal Society, y en los meses que siguen, todas las predicciones del modelo kuhniano de revolución científica se cumplen una tras otra, especialmente el desplazamiento de paradigma ("paradigm shift») y el enrolamiento masivo («bandwagon effect»). Poco después, J. Tuzo Wilson antuncia que se ha producido una revolución en las ciencias de la Tierra, y a principios de los años setenta, la conversión de la comunidad geológica es prácticamente completa. Pero esta comunidad está deslumbrada por las novedades oceanográficas: dorsales, zonas de Benioff, fallas transformantes, puntos calientes oceánicos... durante un tiempo, los continentes son nada más que piezas de un puzzle para encajar unas con otras.

Así, en 1965, uno de los más importantes tectónicos de Francia, Jean Aubouin, podía publicar un gran libro de síntesis que se titula, sencillamente, Geosinclinales, y en 
cuyo prólogo se defendía desdeñosamente de la revolución en marcha:

«...el concepto de geosinclinal es un concepto geológico basado en el estudio e interpretación de series de rocas, por lo cual no es posible rechazarlo en favor de otros fundados en el estudio de fenómenos contemporáneos, no importa que estén basados en estudios oceanográficos o en datos geofísicos».

Se trataba, evidentemente, de la última línea de resistencia. En los años siguientes, las cadenas de montañas se reinterpretan como estructuras producidas por subducción y colisión (Dewey y Bird 1970a, Dewey y Horsfield 1970), y en seguida comienzan a aparecer trabajos (Dewey y Bird 1970b, Hsü 1972, Wang 1972) en los que se intenta una reconversión parcial del concepto de Hall a la Nueva Geología. Quizá Io más representativo de esta fase sea la propuesta con la que Hsú cierra su artículo: mantener el concepto de geosinclinal con una validez restringida, en concreto «...un conjunto tectónico de secuencias sedimentarias y sus rocas asociadas, que se encuentran en los actuales bordes continentales o márgenes de placa».

\section{EL RELEVO (DE 1976 EN ADELANTE)}

La enmienda transaccional de Hsü tuvo un cierto eco: como puede verse en la figura 1 , en 1980 el término «geosinclinal» aparecía más en los títulos de artículos científicos que en 1940, tanto en términos absolutos como relativos. Aunque el autor de esta estadística (Kelling 1981) advierte que en buena parte ello se debe a la influencia de las publicaciones de Europa Oriental, lo cierto es que se podría pensar que, en efecto, el concepto había pasado la prueba y había sido incorpora-

\section{figura 1}

Número y porcentaje de artículos de revistas internacionales de Geología que contienen en su título la palabra "geosinclinal". En Kelling (1981)

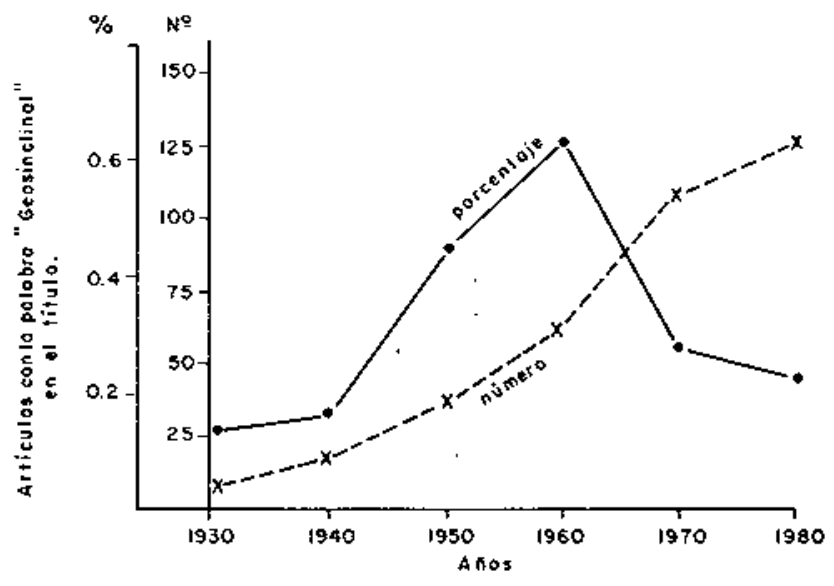

do a la terminología de la Nueva Geología. El mismo Kelling, por ejemplo, concluía que «el concepto de geosinclinal está demasiado incardinado en la literatura geológica para ser descartado de golpe».

Una revisión personal nos ha permitido reevaluar esta idea. En los números publicados en 1989 por cinco revistas internacionales de Geología (Earth and Planetary Science Letters, Nature y Tectonophysics, europeas; y Geology y el Bulletin of the Geological Society of America, norteamericanas) el término «geosinclinal» ya no aparece ni una sola vez, a pesar de que en varios números de Tectonophysics hay un volumen considerable de trabajos firmados por geólogos de la Unión Soviética. Significativamente, tampoco las placas litosféricas aparecen excesivamente en los títulos; pero sí la jerga auxiliar de la tectónica de placas: suturas, oroclinales, márgenes activas, colisiones, «terranes», polos paleomagnéticos, puntos triples, etc. Esto significa que ya no hace falta decir expresamente que un artículo se enmarca en la idea de la movilidad litosférica, porque ello se da por sobreentendido. Kuhn llamó «ciencia normal» a esta situación, en la que las banderas (y banderines de enganche) han vuelto a guardarse hasta la próxima revolucion.

\section{DISCUSIÓN: EL NUEVO PAPEL DE UNA VIEJA IDEA}

¿Por qué se ha producido un relevo tan nítido? Porque la mayoría de los científicos activos en los últimos diez años en el área de las ciencias de la Tierra no han seguido los consejos de cautela, y han preferido posturas más radicales, como la que con gran visión exponía Coney en 1970, refiriéndose a la última etapa de la teoría geosinclinal: «... una característica de esta confusión es el uso de palabras que han perdido su significado, y de modelos que ya no son aplicables. Sugiero que necesitamos una nueva terminología, tanto como un nuevo modelon.

¿Por qué la palabra «geosinclinal» había perdido su significado? Porque, cuando el furor de los datos oceanográficos y geofísicos pasó, los geólogos volvieron a analizar, con otros ojos, las mismas cadenas de montañas que venían siendo interpretadas desde hacía un siglo como resultado de sedimentación, subsidencia, movimientos en la vertical y fases compresivas universales de origen incierto. $Y$, aunque no inmediatamente, comenzaron a ser capaces de reinterpretarlas en términos movilistas. Primero fueron los ejemplos más simples, como los Andes o el Himalaya; luego, otros que requerían colisiones de microplacas y rotaciones, como los Alpes; después, cadenas más antiguas, como el Hercínico y el Caledónico, productos de viejas colisiones bicontinentales; por último, hubo que acuñar nuevos conceptos, como el de «terreno»(1) («terrane») para explicar cadenas, como las Rocosas, producidas por impacto repetido de microcontinentes contra un borde subductivo.

En todas estas cadenas de montañas, el ciclo geotectónico era simplemente inaplicable. En primer Iugar, una 
fuerte sedimentación no era una fase previa indispensable: por ejempio, en la cađena caledónica en Escocia, lo que se interpretaba como una secuencia sedimentaria de $9000 \mathrm{~m}$ de espesor ha resultado ser un prisma de acreción formado por una decena de unidades de grosor moderado, apiladas tectónicamente en una zona de subducción. Ello indica que, además, la deformación no es una segunda fase posterior a la sedimentación, sino que es simultánea con el grueso de ella; $\mathrm{y}$, además, que se produce durante periodos de tiempo mucho más largos que los previstos en el «ciclo geotectónico», y que la moderna geocronología ha permitido precisar: unos 80 millones de años de deformación y metamorfismo $(y, a l$ final, magmatismo) en los Alpes, y una cifra similar para el hercínico español. Estos larguísimos periodos representan en esencia toda la larga colisión e interpenetración bicontinentales que cierran las orogenias, y dejan sin contenido el viejo concepto de «fase orogénica», que a lo sumo puede representar la colisión de un «terreno», y tener por lo tanto un valor sólo regional. Ni siquiera la acomodación de eugeosinclinal y miogeosinclinal a una costa atlántica es sostenible, porque el eugeosinclinal debe contener abundantes rocas volcánicas, y los prismas sedimentarios del pie del talud están desprovistos de ellas; hoy sabemos que las rocas volcánicas se mezclan (tectónicamente) con los sedimentos sólo durante la subducción, en los llamados «mélanges» ofiolíticos (Bond y Kominz 1988).

Existe, por último, una diferencia filosófica fundamentaI entre el concepto de «ciclo geotectónico» y la Geología moderna. El primero es un concepto estrictamente determinista, en el que los acontecimientos se suceden

(I) «Litosferoclasto» es una traducción castellana etimologicamente correcta pero estéticamente lamentable de este término. unos a otros inexorablemente. El vagar de los continentes a través de la superficie del planeta es un proceso mucho más aleatorio, que por ello explica mucho mejor la gran diversidad de las cadenas de montañas que se producen a causa de su colisión. Variables como el ángulo y duración de la subducción, el relieve de la placa subducida, La existencia o no de arcos insulares omicroplacas intermedios, y la misma cinemática del proceso son los rasgos básicos que los geólogos que analizan las cadenas tras la revolución están empezando a desentrañar. Así como los petrólogos de los años cincuenta repetian con Read: «... hay granitos y granitos...», los tectónicos de hoy han Ilegado al liberador convencimiento de que hay colisiones y colisiones...

\section{CONCLUSIÓN: ¿HAY QUE OLVIDAR EL CONCEPTO DE GEOSINCLINAL?}

Rotundamente, no. Sólo hay que limitarse a ponerlo en el lugar que le corresponde, que es la historia de las teorías orogénicas. Afortunadamente, la historia de la ciencia está Ilena de casos semejantes: el flogisto, el éter o los átomos indivisibles son conceptos que han centrado las ideas cientf́ficas durante una época, a veces muy larga, para luego pasar al museo de las ideas que han marcado el trabajoso camino del hombre hacia la comprensión de la Naturaleza. Pero, ¿qué pensaríamos de un libro de Física que metiese en el mismo saco el flogisto y la energía nuclear, el éter y el espacio-tiempo curvo, los átomos indivisibles y los quarks...?

\section{REFERENCIAS BIBLIOGRÁFICAS}

AUBOUIN, J., 1965. Geosynclines. (Elsevier: Amsterdam). $335 \mathrm{pp}$.

BOND, G.C. y KOMINZ, M.A., 1988. Evolution of thought on passive continental margins from the origin of geosynclinal theory (1860) to the present, Geol. Soc. Am. Bull, 100, pp. 1909-1933.

CONEY, P.J., 1970. The geotectonic cycle and the new global tectonics, Geol. Soc. Am. Bull., 81, pp. 739-748.

DEWEY, J.F. y BIRD, J.M., 1970a. Mountain belts and the new global tectonics, J. Geophys. Res, 75, pp. 2625-2647.

DEWEY, J.F. y BIRD, J.M., 1970b. Plate tectonics and geosynclines, Tectonophysics, 10 , pp. 625-638.

DEWEY, J.F. y HORSFIELD, B., 1970. Plate tectonics, orogeny and continental growth, Nature, 225, pp. 521-525.
DIETZ, R.S., 1963. Collapsing continental rises: an actualistic concept of geosynclines and mountain building, J. Geol., 71, pp. 314-333.

HSÜ, H. J., 1972. The concept of the geosyncline, ycsterday and today, Trans. Leicester Lit. Phil. Soc., 66, pp. 26-48.

KAY, M. 1951. North American geosynclines. Memoir 48, Geol. Soc. Am., 143 pp.

KELLING, G., 1981. Is the geosyncline dead?, G. Teaching, 6, pp. 117-123.

KUHN, T.S., 1962 [1971]. La estructura de las revoluciones científicas. (Fondo de Cultura Económica: Madrid), 320 pp.

WANG, C.S., 1971. Geosynclines in the new global tectonics, Geol. Soc. Am. Bull., 83, pp. 2105-2110. 